\title{
Plasma Production via Field Ionization
}

\author{
C.L. O'Connell, C.D. Barnes, F.-J. Decker, M.J. Hogan, R. Iverson, P. Krejcik, R. Siemann, D.R. Walz \\ SLAC, Menlo Park, CA 94025, USA
}

\author{
C.E. Clayton, C. Huang, D.K. Johnson, C. Joshi, W. Lu, K.A. Marsh, W. Mori, M. Zhou \\ UCLA, Los Angeles, CA 90095, USA
}

\author{
S. Deng, T. Katsouleas, P. Muggli, E. Oz \\ USC, Los Angeles, CA 90089, USA
}

(Dated: October 4, 2006)

\begin{abstract}
Plasma production via field ionization occurs when an incoming particle beam is sufficiently dense that the electric field associated with the beam ionizes a neutral vapor or gas. Experiments conducted at the Stanford Linear Accelerator Center explore the threshold conditions necessary to induce field ionization by an electron beam in a neutral lithium vapor. By independently varying the transverse beam size, number of electrons per bunch or bunch length, the radial component of the electric field is controlled to be above or below the threshold for field ionization. Additional experiments ionized neutral xenon and neutral nitric oxide by varying the incoming beam's bunch length. A self-ionized plasma is an essential step for the viability of plasma-based accelerators for future high-energy experiments.

PACS numbers: 41.75.Lx, 41.75.Ht, 52.40.Mj, 52.50.-b
\end{abstract}

\section{INTRODUCTION}

The successful demonstration of a beam-driven plasma wake field accelerator (PWFA) over macroscopic distances is a critical milestone in the progression of plasmas from laboratories to future high-energy accelerators and colliders, where a combination of high density and long length will be required. In a paper recently published on experiments performed at the Stanford Linear Accelerator Center (SLAC), a beam-driven PWFA showed accelerating gradients of greater than $30 \mathrm{GeV} / \mathrm{m}$ over a $10 \mathrm{~cm}$-length plasma, which were achievable due to the incoming beam's ability to simultaneously ionize a neutral $\mathrm{Li}$ vapor and drive a large-amplitude wake to accelerate the tail particles [1].

These experiments were performed in a non-linear, relativistic regime and, based on simulations, the accelerating gradient of the system in this regime increases as the bunch length decreases; consequently, ultra-short bunches are preferred [2]. When a high-density, ultrashort bunch enters a region filled with a neutral vapor or gas, the electric field associated with the beam can ionize the valence electron of each neutral atom in its vicinity leaving a fully ionized plasma for the remainder of the bunch [3]. Lithium (Li) has a relatively low ionization potential for the first electron $(5.4 \mathrm{eV})$, which allows ionization over a broad range of beam parameters. The larger ionization potential of the second electron $(75.6 \mathrm{eV})$ ensures the plasma density does not evolve significantly along the bunch due to secondary ionization. Xenon (Xe) and nitric oxide (NO) also have relatively low ionization potentials for the first electron, $12.13 \mathrm{eV}$ and $9.25 \mathrm{eV}$, respectively, and are, therefore, possible alternatives to $\mathrm{Li}$.

After the beam ionizes the vapor, it expels the plasma electrons due to its space-charge field and generates its own plasma wake. Consequently, the field ionization is detected and measured by the beam's energy loss through the vapor column due to plasma wake production [4]. The plasma ions, which are far more massive than the plasma electrons, remain stationary during the time scale of the beam passing through the plasma and exert a restoring force on the plasma electrons. This leads to a plasma electron oscillation and results in a plasma electron density spike on axis, which creates a high-gradient accelerating structure with a wavelength set by the plasma density.

When the plasma wake and bunch length are properly matched, the electric field associated with the density spike of plasma electrons accelerates the back end of the electron beam. For the correct combination of bunch length and vapor density, the beam's electric field can ionize a neutral vapor thereby generating its own plasma and the resulting space-charge field drives a highamplitude wake to accelerate the beam's tail particles.

\section{FIELD IONIZATION THEORY}

The most widely accepted approximation of the field ionization rate is the Ammosov, Delone and Krainov formula for atoms in an alternating electric field [5]. The ADK theory is a fully generalized expression for the field ionization rate of a complex atom in an arbitrary state, which is described by an effective quantum number, $n^{*}=Z / \sqrt{2 \varepsilon_{0}}$, where $Z$ is the charge of the atomic residue (e.g. one for first ionization and two for secondary ionization) and $\varepsilon_{0}$ is the ionization energy. The ADK rate is either time averaged over a laser cycle or it can be written as an instantaneous rate due to the beam's local

\section{Submitted to Phys.Rev.ST Accel.Beams}


electric field. The instantaneous ADK tunneling rate, in atomic units, is:

$$
\begin{aligned}
W_{A D K}= & C_{n^{*} \ell}^{2} f(\ell, m)\left|\varepsilon_{0}\right|\left(\frac{2}{E}\left(2\left|\varepsilon_{0}\right|\right)^{3 / 2}\right)^{2 n^{*}-|m|-1} \\
& \times \exp \left(-\frac{2\left(2\left|\varepsilon_{0}\right|\right)^{3 / 2}}{3 E}\right)
\end{aligned}
$$

where $E$ is the beam's radial electric field and $C_{n^{*} \ell}$ and $f(\ell, m)$ are defined as

$$
\begin{aligned}
& C_{n^{*} \ell}=\left(\frac{2 e}{n^{*}}\right)^{n^{*}}\left(2 \pi n^{*}\right)^{-1 / 2}, \\
& f(\ell, m)=\frac{(2 \ell+1)(\ell+|m|) !}{2^{|m|}|m| !(\ell-|m|) !} .
\end{aligned}
$$

The constant $e$ in the coefficient $C_{n^{*} \ell}$ is Euler's number 2.718 and $\ell$ and $m$ are the angular and magnetic quantum numbers, respectively. The validity of the ADK formula and, specifically, $C_{n^{*} \ell}$ is expected to be best in the quasi-classical approximation, $n^{*} \gg 1$; however, the approximation is accurate to a few percent up to values of $n^{*} \approx 1$ based on numerical calculations of the coefficient, $C_{n^{*} \ell}[5]$.

\section{EXPERIMENTAL APPARATUS AND TECHNIQUES}

A single $28.5 \mathrm{GeV}$ electron bunch from the linear accelerator enters the Final Focus Test Beam (FFTB) facility at a rate of either 1 or $10 \mathrm{~Hz}$. Figure 1 illustrates the primary features of the experimental setup. Upon entering the FFTB, the beam traverses a weak vertical chicane located within a high horizontal dispersion region and emits synchrotron radiation. The X-ray portion of the synchrotron spectrum scintillates in a cerium doped yttrium aluminum garnet (Ce:YAG) screen and is imaged on a charge coupled device (CCD) camera. This allows for a non-destructive determination of the beam's incoming energy spread $[1,6]$. Prior to reaching the plasma source, the beam traverses two $1 \mu \mathrm{m}$ thick titanium $(\mathrm{Ti})$ foils and generates transition radiation through both. On the upstream foil, which is located $20 \mathrm{~m}$ upstream of the plasma source, a pyroelectric detector monitors the coherent transition radiation (CTR) energy. At wavelengths longer than the bunch length, the transition radiation becomes coherent and provides a shot-to-shot measurement of the relative bunch length, where the integrated CTR energy increases as the bunch length decreases. Downstream of the CTR foil and about $1 \mathrm{~m}$ upstream of the plasma, a CCD camera captures the optical transition radiation (OTR) to profile the transverse component of the beam before it enters the plasma. The transverse profile is then used for tuning the linear accelerator. Downstream of the plasma, the beam passes through a third and final $1 \mu \mathrm{m}$-thick Ti foil, where a

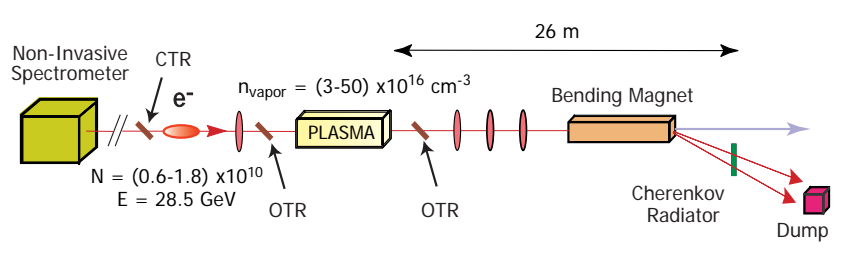

FIG. 1: Schematic of the E164 experimental layout. The diagram is not to scale.

CCD camera monitors the OTR and provides information about plasma focusing and possible deflection [7].

Before the beam reaches the plasma, it is focused transversely in order to minimize the beam size at the plasma entrance. Depending on the experiment, the beam then enters one of two types of plasma sources: a heat-pipe oven [8] or a gas cell. In the case of the Li heat-pipe oven, the vapor region varies in length between $6-10 \mathrm{~cm}$, with a vapor density of $(3-25) \times 10^{16} \mathrm{~cm}^{-3}$. The design of the heat-pipe oven results in a section on either side of the oven, where the Li density profile transitions from zero to full density and is typically on the order of $10 \mathrm{~cm}$ for these experiments. To allow for plasma-off cases, the oven is placed on a pneumatic mover that moves the oven in and out of the beam path.

Additional experiments were performed with a gas cell filled with either Xe $\left(\varepsilon_{0}=12.13 \mathrm{eV}\right)$ or $\mathrm{NO}\left(\varepsilon_{0}=\right.$ $9.25 \mathrm{eV}$ ). Although NO is a diatomic molecule, previous experiments performed by Walsh et al in 1993, show that NO was ionized before dissociating [9]. Then in 1994, Walsh et al also showed that the ionization rate of the first electron in NO agreed well with the quasi-static tunneling model described by the ADK theory [10]. The gas cell is an appealing alternative to the heat-pipe oven since it provides sharp boundaries and the cell can attain much higher vapor densities than can be sustained in the heatpipe oven. Unlike the heat-pipe oven, which takes hours to stabilize after significant changes, the gas cell allows for instantaneous changes to vapor density and column length. The gas cell has a variable length of $2.2-11 \mathrm{~cm}$ and the density, which is solely dependent on the gas pressure inside the cell, varies from $(1-15) \times 10^{17} \mathrm{~cm}^{-3}$. For the plasma-off cases, the chamber is filled with helium $\left(\varepsilon_{0}=24.6 \mathrm{eV}\right)$, which the beam cannot ionize given its parameters for these particular experiments.

Beyond the plasma source, dipole magnets vertically disperse the bunch in energy while quadrupole magnets image the bunch exiting the plasma onto a $1 \mathrm{~mm}$-thick piece of fused silica aerogel located $25 \mathrm{~m}$ downstream. The resulting Cherenkov light is collected and imaged onto a CCD camera, where the vertical axis of the image is dominated by the beam's energy spread and the horizontal axis is the transverse size of the beam. The imaging spectrometer is crucial for differentiating plasmainduced energy changes to the beam from possible transverse deflections caused by the strong focusing forces of 
TABLE I: Typical beam and plasma parameters

Number of $\mathrm{e}^{-}$per bunch

Bunch Energy

Bunch Length $[\mu \mathrm{m}]$

Transverse Beam Size $[\mu \mathrm{m}]$

Vapor Density $\left[\mathrm{cm}^{-3}\right]$

Plasma Oven Length [cm]

Oven Transition Length $[\mathrm{cm}]$

Gas Cell Length [cm]
$(0.6-1.82) \times 10^{10}$

$28.5 \mathrm{GeV}, 5.6 \times 10^{4}$

$20-110$

$10-55$

$(3.0-50.0) \times 10^{16}$

$6-10$

$7-10$

$2.2-11$

the ion channel. See Table I for the typical beam and plasma parameter ranges.

Although no diagnostic directly measures the current profile of the compressed electron bunch entering the plasma, it is possible to measure it indirectly. Within reasonable ranges of accelerator parameters that affect the bunch compression process, the energy spectrum measured at the end of the linac is unique. By using the 2-D $\left(z, p_{z}\right)$ simulation code LITRACK [11] to match the energy spectra measured in the FFTB with simulated ones, we are able to infer the incoming phase space and profile.

Using one of the data runs presented as an example, Fig. 2 illustrates the beam's phase space and the resulting longitudinal charge profile output from LITRACK. Note that in addition to the central Gaussian-shaped distribution in the charge profile, it also has non-Gaussian wings. The bunch length quoted in the text reflects only the central Gaussian distribution. All the bunch lengths presented in this paper are based on results produced by the LITRACK simulations and any calculations that rely on those bunch lengths scales the beam's total charge such that the peak current is consistent between the experimental conditions and the Gaussian approximations.

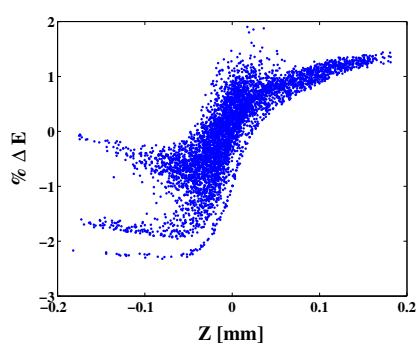

(A)

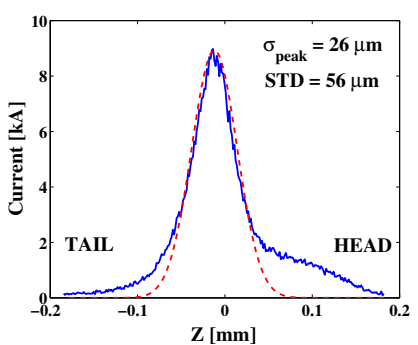

(B)

FIG. 2: (A) An example of the incoming beam's phase space according to LITRACK simulations. (B) Longitudinal charge profile of the incoming beam obtained by the projection of the phase space from (A). The mathematical standard deviation of the entire distribution is $56 \mu \mathrm{m}$ and the standard deviation of the central Gaussian-shaped distribution, plotted with the dashed line, is $26 \mu \mathrm{m}$.

TABLE II: Data Run Experimental Conditions

\begin{tabular}{l||lr|lr}
\hline \hline $\mathrm{Li}-\Delta \sigma_{r}$ & $N$ & $(0.87-0.90) \times 10^{10}$ & $n_{v}$ & $3 \times 10^{16} \mathrm{~cm}^{-3}$ \\
& $\sigma_{r}$ & $10-50 \mu \mathrm{m}$ & $L$ & $10 \mathrm{~cm}$ \\
$\sigma_{z}$ & $32 \mu \mathrm{m}$ & $L_{T}$ & $7 \mathrm{~cm}$ \\
$\mathrm{Li}-\Delta N$ & $N$ & $(0.60-1.43) \times 10^{10}$ & $n_{v}$ & $20 \times 10^{16} \mathrm{~cm}^{-3}$ \\
& $\sigma_{r}$ & $20 \mu \mathrm{m}$ & $L$ & $6 \mathrm{~cm}$ \\
$\mathrm{Li}-\Delta \sigma_{z}$ & $\sigma_{z}$ & $26 \mu \mathrm{m}$ & $L_{T}$ & $8 \mathrm{~cm}$ \\
& $N$ & $(0.87-0.89) \times 10^{10}$ & $n_{v}$ & $3 \times 10^{16} \mathrm{~cm}^{-3}$ \\
$\sigma_{r}$ & $15 \mu \mathrm{m}$ & $L$ & $10 \mathrm{~cm}$ \\
$\mathrm{Xe}-\Delta \sigma_{z}$ & $\sigma_{z}$ & $25-105 \mu \mathrm{m}$ & $L_{T}$ & $7 \mathrm{~cm}$ \\
& $N$ & $(1.72-1.82) \times 10^{10}$ & $n_{v}$ & $9.9 \times 10^{16} \mathrm{~cm}^{-3}$ \\
$\mathrm{NO}-\Delta \sigma_{z}$ & $\sigma_{r}$ & $15 \mu \mathrm{m}$ & $L_{G}$ & $9.2 \mathrm{~cm}$ \\
$\sigma_{z}$ & $20-60 \mu \mathrm{m}$ & & \\
& $N$ & $(1.63-1.72) \times 10^{10}$ & $n_{v}$ & $13 \times 10^{16} \mathrm{~cm}^{-3}$ \\
$\sigma_{r}$ & & $15 \mu \mathrm{m}$ & $L_{G}$ & $8 \mathrm{~cm}$ \\
$\sigma_{z}$ & $26-42 \mu \mathrm{m}$ & & \\
\hline \hline
\end{tabular}

\section{EXPERIMENTAL RESULTS}

The rate of field ionization is related to the local electric field associated with the incoming bunch. In this paper, we use the peak radial electric field to indicate whether the threshold for field ionization is crossed. The maximum electric field for an incoming electron bunch, which is Gaussian along the radial and longitudinal components, can be conveniently summarized into the following engineering formula:

$$
E_{\text {peak }} \approx 10.4 \frac{\mathrm{GV}}{\mathrm{m}}\left[\frac{N}{1 \times 10^{10}}\right]\left[\frac{10}{\sigma_{r}(\mu m)}\right]\left[\frac{50}{\sigma_{z}(\mu m)}\right],
$$

where $N$ is the number of electrons per bunch, $\sigma_{r}$ is the RMS value of the transverse beam size in $\mu \mathrm{m}$ and $\sigma_{z}$ is the RMS value of the beam's bunch length in $\mu \mathrm{m}$. By independently varying each one of these three components, the radial electric field is controlled to be above or below the threshold for field ionization. Without plasma production, the beam would pass through the vapor without any interaction, as if it were a drift section.

For reference, Table II compiles the specific experimental conditions for each of the data runs described in this section. In the table notation, $\mathrm{Li}-\Delta \sigma_{r}$ refers to section A.1, Li $-\Delta N$ refers to section A.2, Li - $\Delta \sigma_{z}$ refers to section A.3, Xe $-\Delta \sigma_{z}$ refers to section B and NO $-\Delta \sigma_{z}$ refers to section $\mathrm{C}$

\section{A. Lithium}

\section{Changing Transverse Beam Size $\left(\sigma_{r}\right)$}

According to Eq. 2, the peak electric field is inversely proportional to the transverse beam size of the incoming bunch. Holding the other two variables $\left(N\right.$ and $\left.\sigma_{z}\right)$ constant and varying the incoming beam's transverse size, 
the electric field is increased until the field ionization threshold is crossed. To observe the transverse beam size threshold, the waist location for the incoming beam was varied along the beam line using the two quadrupoles upstream of the plasma entrance. For the data set described, the waist was moved from $45 \mathrm{~cm}$ upstream of the plasma entrance to $55 \mathrm{~cm}$ downstream of the plasma entrance, in $5 \mathrm{~cm}$ increments with 10 events taken at each step.

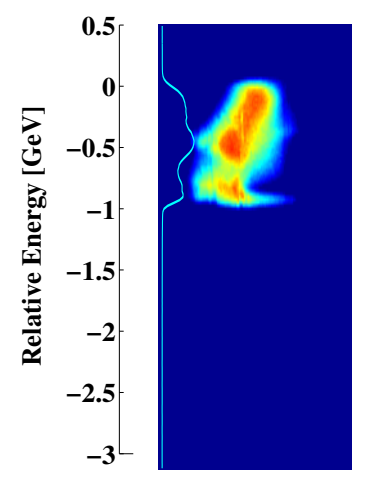

(A)

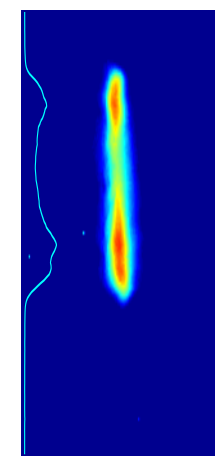

(B)

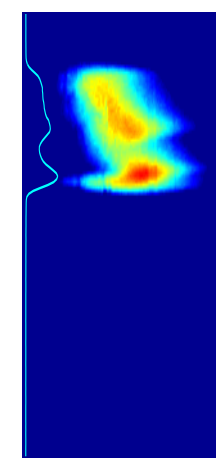

(C)
FIG. 3: All images show the energy spectrum of the electron beam at the Cherenkov radiator. The scale on the left indicates the relative energy along the vertical component, where zero is defined to be the highest energy without plasma. The images are plotted with a logarithmic color map in order to bring out the tails. The vertical profile is superimposed along the left edge of the image. The three events are taken over a period of several minutes. (A) The beam's waist located $45 \mathrm{~cm}$ upstream of the plasma entrance and no ionization has occurred in the $\mathrm{Li}$ vapor. (B) The waist at the plasma entrance and ionization of the $\mathrm{Li}$ vapor. (C) The waist located $55 \mathrm{~cm}$ downstream of the plasma entrance and again no ionization of the $\mathrm{Li}$ vapor.

As discussed in the introduction, the field ionization effects are characterized by the significant amount of energy loss observed once the incoming beam's density surpasses the field ionization threshold. Figure 3 illustrates that energy loss using the energy spectrum of the bunch at the Cherenkov diagnostic after exiting the plasma at three waist locations. Recall that the beam at the Cherenkov radiator is dominated by energy spread along the vertical axis, where the more energetic particles are at the top of the image, and the horizontal axis is the transverse size of the beam. The image on the far left is the waist pulled upstream of the plasma entrance and no ionization occurs. For the center image the waist is located at the plasma entrance and the transverse size is small enough the electric field ionizes the vapor. When the waist is located downstream of the plasma entrance, the beam density is again insufficient to ionize the vapor, as is seen in the image on the far right. The beta function with the beam's waist at the plasma entrance is approximately $10 \mathrm{~cm}$ in $x$ or the horizontal plane and $1 \mathrm{~cm}$ in

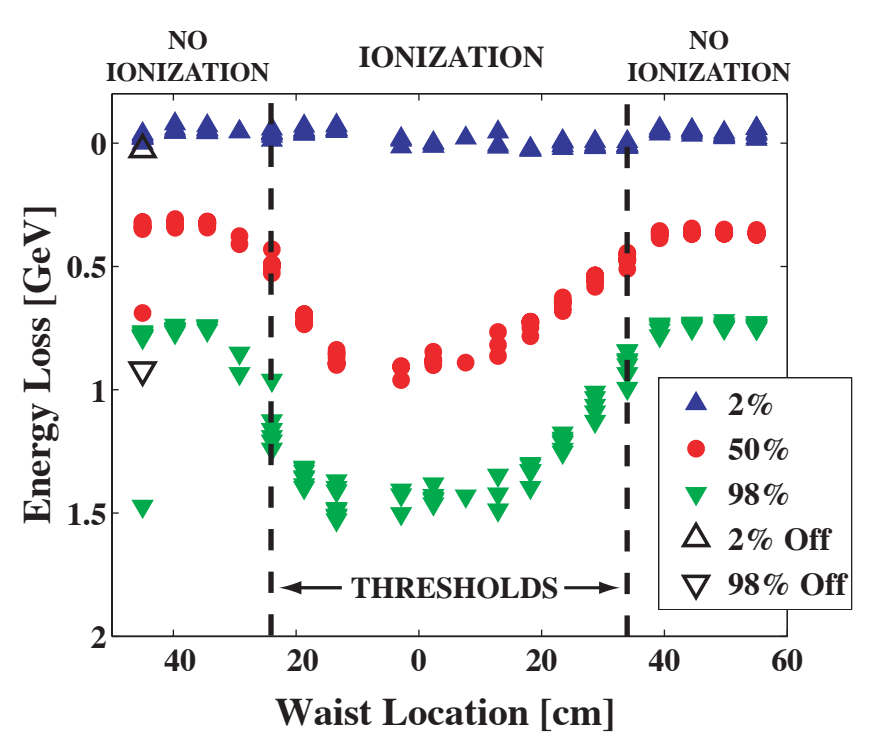

FIG. 4: $\left(\mathrm{Li}-\Delta \sigma_{r}\right)$ Distribution of the $2 \%, 50 \%$ and $98 \%$ charge levels as a function of the beam's waist location. The $\triangle$ and $\nabla$ represent the $2 \%$ and $98 \%$ charge levels of the beam with no plasma. For illustration, the ionization threshold is noted by the dashed lines.

$y$ or the vertical plane, due to the flat beam $\left(\epsilon_{x} \gg \epsilon_{y}\right)$ configuration of the linear accelerator.

The change in beam size from the smallest with the waist at the plasma entrance and to the largest with the waist pulled $55 \mathrm{~cm}$ downstream is from about $10 \mu \mathrm{m}$ up to approximately $50 \mu \mathrm{m}$ in $x$. Since the betas have an order of magnitude difference along the $x$ and $y$ components, the transverse size only significatly varies along the $x$-axis for small changes in the waist location.

Figure 4 graphically displays the charge distribution at the Cherenkov detector as a function of waist location. Three distribution levels are plotted: $2 \%, 50 \%$ and $98 \%$. The $2 \%(50 \%, 98 \%)$ level is defined as the energy where $2 \%(50 \%, 98 \%)$ of the charge is located at a higher energy. For reference, the $2 \%$ and $98 \%$ distribution levels with the plasma oven removed from the beam path are also plotted. The average energy of the beam is defined as $50 \%$ distribution level, while the peak energy of beam is illustrated by the $98 \%$ distribution level. The change in average energy between the non-ionizing case and the maximum ionization is defined as $\Delta E_{a v g}$. The change in peak energy between the two cases is defined as $\Delta E_{\text {peak }}$. Between the waist at the plasma entrance and the waist at $-30 \mathrm{~cm}, \Delta E_{a v g}$ was $650 \mathrm{MeV}$ and $\Delta E_{\text {peak }} 800 \mathrm{MeV}$. As is seen in Fig. 4, the ionization threshold is crossed at a waist location of around $-28 \mathrm{~cm}$ or a transverse size of $21 \mu \mathrm{m}$, based on previous measurements with a wire scanner along the $x$-component as a guide [4].

In order to limit the fluctuations due to changing charge and changing bunch length, we chose a subset of the data based on the incoming charge and on the CTR diagnostic, which is inversely proportional to the 


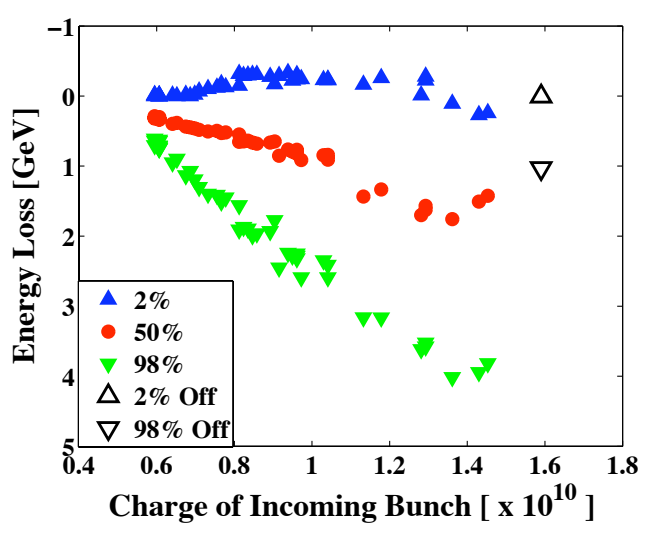

FIG. 5: $(\mathrm{Li}-\Delta N)$ Distribution of the $2 \%, 50 \%$ and $98 \%$ charge levels as a function of the incoming bunch charge. The $\triangle$ and $\nabla$ represent the $2 \%$ and $98 \%$ charge levels of the beam with no plasma.

beam's bunch length. The charge was limited to be between $0.87 \times 10^{10}$ and $0.90 \times 10^{10}$ electrons per bunch, the $E_{C T R}$ signal was restricted to a range which translated to a bunch length of around $32 \mu \mathrm{m}$ for the center Gaussian distribution, according to LITRACK. These limits on charge and CTR energy reduced the data set from 200 events down to 103. Based on the beam parameters, the peak electric field associated with the threshold conditions was $5.25 \mathrm{GV} / \mathrm{m}$.

\section{Changing Bunch Charge (N)}

Again referring to Eq. 2, the peak electric field is proportional to the number of electrons in the incoming bunch. Holding the other variables constant and varying the incoming beam's charge from $0.60 \times 10^{10}$ to about $1.43 \times 10^{10}$ electrons per bunch, the electric field is increased until the field ionization threshold is crossed.

Figure 5 graphically displays the charge distribution at the Cherenkov detector as a function of the number of electrons in the incoming bunch. Once again, the $2 \%$ and $98 \%$ distribution levels with no plasma are also plotted at high charge. Between lowest and highest charges for the data set, $\Delta E_{\text {avg }}$ was $1.4 \mathrm{GeV}$ and $\Delta E_{\text {peak }}$ was about $3.5 \mathrm{GeV}$.

The data for Fig. 5 was acquired at a rate of $1 \mathrm{~Hz}$ over a period of two hundred seconds. In order to limit variations in the incoming bunch length, we restricted the data set based on the CTR diagnostic. The signal of that diagnostic, $E_{C T R}$, is proportional to $N^{2} / \sigma_{z}$. Since the charge of the incoming bunch was varied, a cut was placed on $E_{C T R} / N^{2}$, to ensure a consistent bunch length. This reduced the data from 200 events down to 47. The LITRACK simulation code was used to determine the bunch length for the remaining events, which was approximately $26 \mu \mathrm{m}$ for the center peak of the longitudinal charge distribution. The transverse beam size was around $20 \mu \mathrm{m}$ and any variations in the beam size are related to changes in the emittance associated with changing the charge and the inherent jitter of the twomile long linear accelerator. Using the images from the upstream OTR to examine the emittance jitter of the transverse beam size shows the shot-to-shot jitter is too small to affect the ionization threshold but as the beam's charge increased, the transverse beam broadened horizontally. This broadening would have a small affect on the ionization threshold because the incremental change is minimal, however, the larger transverse beam size at high charge will result in less energy loss than would be expected if it remained constant.

The ionization threshold was crossed around $0.65 \times 10^{10}$ electrons per bunch. The threshold is not as pronounced due to the fact that the energy loss measurement is an indirect measurement of the ionization and changes in the beam's charge also implies changes in the energy spectrum. Unlike changes to the beam's transverse size, which occur immediately upstream of the experimental set-up, variation of the beam's charge occurs at the beginning of the linac, which can subtly alter the incoming energy spectrum. The beam parameters at the ionization threshold translate to a peak electric field of $5.08 \mathrm{GV} / \mathrm{m}$.

\section{Changing Bunch Length $\left(\sigma_{z}\right)$}

The same analysis is repeated by changing the bunch length, while holding the charge of incoming bunch and its transverse beam size constant. In this case, as the bunch length decreases, the electric field increases and is eventually sufficient to ionize the $\mathrm{Li}$ vapor. The signal from the CTR diagnostic is used to sort the data according to the relative bunch length, where an increasing signal indicates a decreasing bunch length.

Figure 6 plots the charge distribution of the beam at the Cherenkov diagnostic as a function of the CTR energy. Between the highest CTR energy (shortest bunch length) produced and the non-ionizing case, $\Delta E_{a v g}$ was almost $800 \mathrm{MeV}$ and $\Delta E_{\text {peak }}$ was approximately $1.2 \mathrm{GeV}$.

The data set was acquired at a rate of $1 \mathrm{~Hz}$ over a period of two hundred seconds. By restricting our analysis to the events with charge between $0.87 \times 10^{10}$ and $0.89 \times 10^{10}$ electrons per bunch, we reduced the data set from 200 events down to 122. According to LITRACK, the bunch length was varied from around $105 \mu \mathrm{m}$ down to $25 \mu \mathrm{m}$. The entire data set was taken with the waist at the plasma entrance with a beam size of around $15 \mu \mathrm{m}$, therefore, any variation in beam size is again due to the inherent emittance jitter associated with the machine.

The field ionization threshold occurs around a CTR energy count of 29 , where a count of zero is equal to the diagnostic reading without any beam. This CTR energy corresponds to a bunch length of $66 \mu \mathrm{m}$ as determined by the LITRACK simulation in conjunction with the X-Ray diagnostic upstream of the experiment. Previous experiments show the CTR energy and the peak current as 


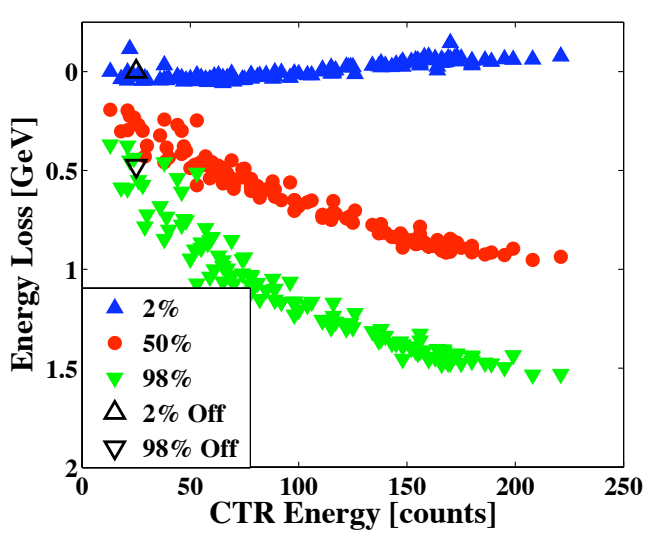

FIG. 6: $\left(\mathrm{Li}-\Delta \sigma_{z}\right)$ Distribution of the $2 \%, 50 \%$ and $98 \%$ charge levels as a function of increasing CTR energy or decreasing bunch length. The $\triangle$ and $\nabla$ represent the $2 \%$ and $98 \%$ charge levels of the beam with no plasma.

determined by LITRACK are well correlated [6]. Because the ionization is exponentially dependent on the local electric field, these systematic errors in determining the bunch length result in non-linear effects on the threshold calculation. Again the threshold is not as pronounced because changes in the CTR energy also implies changes in the energy spectrum which washes out the threshold. The resulting peak electric field at the ionization threshold is $4.08 \mathrm{GV} / \mathrm{m}$.

\section{B. Xenon}

By varying the beam's bunch length, additional experiments on the field ionization threshold were performed using $\mathrm{Xe}\left(\varepsilon_{0}=12.13 \mathrm{eV}\right)$. Figure 7 measures the energy spectrum of the bunch after the gas cell in the case of no ionization and the ionization threshold crossed. The profile for each image is superimposed on the left. Note, in the ionizing case the bulk of charge remains unaffected and there is only a wispy tail with little charge at low energy. This is the result of ionization occurring late in the bunch. There is also a visible increase in X-ray hits, so a median software filter was added to the images to reduce the noise without compromising the data. Median filtering is a nonlinear operation often used in image processing to reduce "salt and pepper" noise. However, the filter was unable to completely eliminate the noise.

Figure 8 plots the three distribution levels of the charge registered at the Cherenkov diagnostic as a function of the CTR energy. The CTR energy value for the Xe data is not comparable to the Li data because of changes in the experimental set-up between the different data sets. The large amount of variation seen in the $98 \%$ level (or peak energy loss) is a combination of two factors. First, the incoming beam's density is not consistently high enough to field ionize the Xe gas, since the smallest bunch length was only barely sufficient to cross the

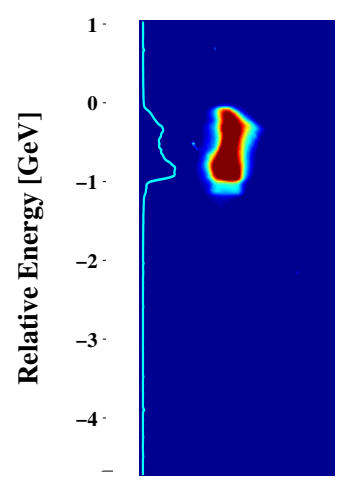

(A)

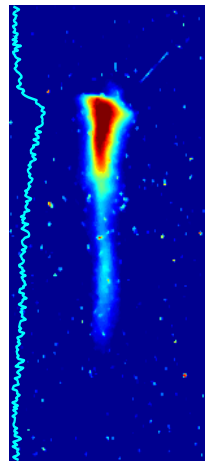

(B)
FIG. 7: Both images show the energy spectrum of the electron beam at the Cherenkov radiator. (A) The bunch is approximately $60 \mu \mathrm{m}$ long and no ionization has occurred in the Xe gas. (B) The bunch is approximately $20 \mu \mathrm{m}$ long and ionizes the Xe gas.

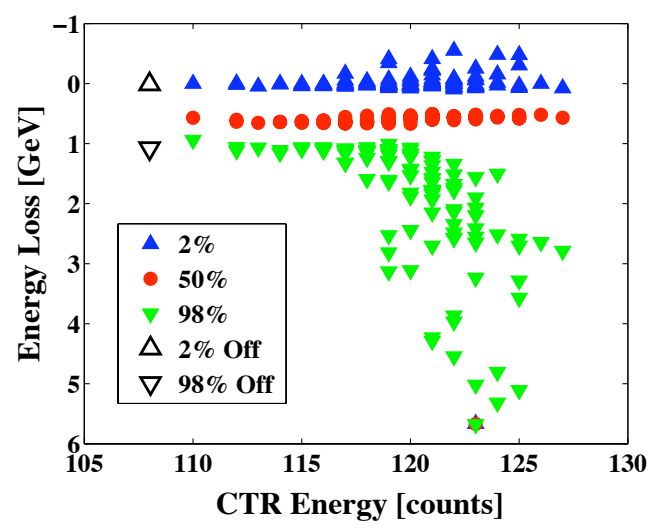

FIG. 8: Changing Bunch Length (Xe) - Distribution of the $2 \%, 50 \%$ and $98 \%$ charge levels as a function of increasing CTR energy or decreasing bunch length.

ionization threshold. Second, the large background noise on the Cherenkov images contributes to the jitter for the extremely large energy loss events for which the number of counts in the low energy region of the image is small.

The data set was composed of 200 events acquired at $1 \mathrm{~Hz}$. The charge of the incoming bunch was between $1.72 \times 10^{10}$ and $1.82 \times 10^{10}$ and, according to LITRACK, the bunch length was varied from $20 \mu \mathrm{m}$ to $60 \mu \mathrm{m}$. To ensure a consistent gas density of Xe, the pressure was maintained at around 3 Torr, which translates into a density of $9.9 \times 10^{16} \mathrm{~cm}^{-3}$. These cuts reduced the data set from 200 events down to 116. At the shortest bunch length, the beam lost a negligible amount of average energy because of ionization occurring late in the bunch, since the bulk of the charge does not lose energy as a result of the wake generation. The peak energy loss is around $2 \mathrm{GeV}$. 


\section{Nitric Oxide}

Since maintaining an incoming beam with sufficient density to repeatedly ionize Xe proved too difficult, NO replaced the Xe in hopes of improving the ionization rate, since NO has a lower ionization potential of $9.25 \mathrm{eV}$ when compared to Xe.

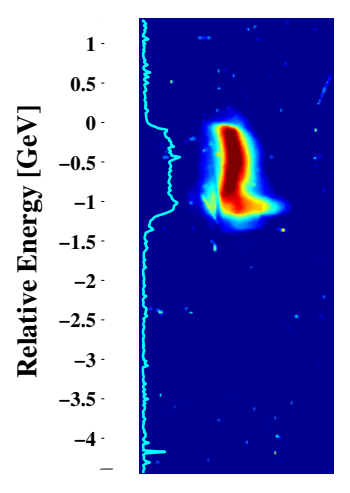

(A)

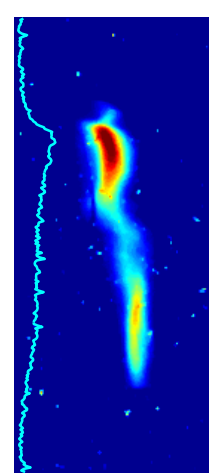

(B)
FIG. 9: Both images show the energy spectrum of the electron beam at the Cherenkov radiator. (A) The bunch is approximately $42 \mu \mathrm{m}$ long and no ionization has occurred in the NO gas. (B) The bunch is approximately $26 \mu \mathrm{m}$ long and ionizes the NO gas.

Figure 9 shows the energy spectrum of the bunch after the gas cell filled with NO in the cases of no ionization and the ionization threshold crossed from a run that varied the incoming bunch length. The same software filter, which was used in the Xe run, was added to the images to reduce the noise without compromising the data. Although NO proved to be more successful than $\mathrm{Xe}$, ionization still occurred too late in the bunch for the accelerating wake to be recovered. Late ionization gives rise to the wispy aspect of the energy loss signal in the $\mathrm{NO}$ and Xe cases, which is seen in Figures 7(B) and 9(B), when compared to the case of $\mathrm{Li}$ for which ionization occurs early in the bunch, see Figure 3(B) for an example. Additionally, the downstream foil of the gas cell was easily damaged when the incoming beam ionized the gas and created plasma. Since NO is a toxic gas and the foils had to be replaced often, the drawbacks of a NO gas cell outweighed any benefits when compared to Xe.

The data set plotted in Fig. 10 was composed of 200 events acquired at $1 \mathrm{~Hz}$. Again, the CTR energy is a relative measurement and not comparable to the other data sets because of changes to the experimental setup. The charge of the incoming bunch was between $1.63 \times 10^{10}$ and $1.72 \times 10^{10}$ and, according to LITRACK, the bunch length was varied from $26 \mu \mathrm{m}$ to $42 \mu \mathrm{m}$. To minimize the amount of background noise on the Cherenkov images, we eliminated very short bunches and, consequently, the images with the highest energy loss and background X-ray

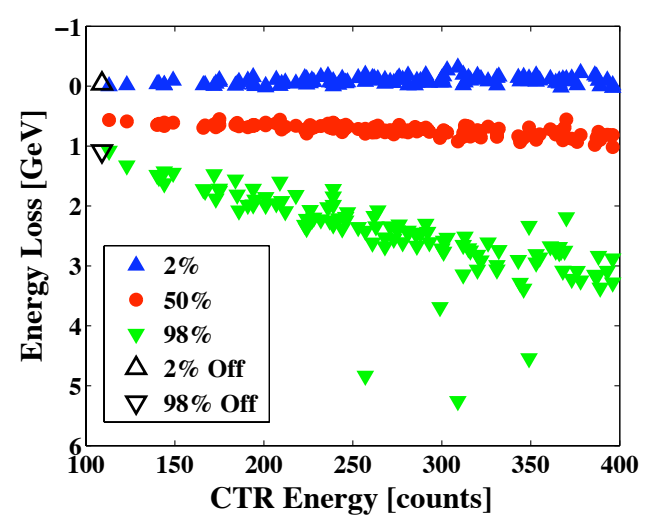

FIG. 10: Changing Bunch Length (NO) - Distribution of the $2 \%, 50 \%$ and $98 \%$ charge levels as a function of increasing CTR energy or decreasing bunch length.

radiation. This reduced the 200 events down to 116 . The gas pressure was maintained at around 4 Torr, a density of $1.3 \times 10^{17} \mathrm{~cm}^{-3}$.

At the shortest bunch length, $\Delta E_{\text {avg }}$ was approximately $400 \mathrm{MeV}$ and $\Delta E_{\text {peak }}$ was $2.2 \mathrm{GeV}$. Some images reached a peak energy loss of greater than $4 \mathrm{GeV}$, without excessive background noise; however, those events were sporadic rather than part of the trend.

\section{CALCULATIONS OF IONIZATION THRESHOLD FOR Li}

Since Eq. 1 assumes a longitudinal and transverse Gaussian distribution, the experimental conditions are approximated to compare the data with the ADK theory. As previously discussed, the charge for the incoming bunch must be scaled such that the peak current is the same for the experiment and the LITRACK results when calculating the fractional ionization.

Table III compares the beam parameters at the ionization threshold for the Li data, the peak electric field associated with those beam conditions and the peak fractional ionization $\left(F_{\text {peak }}\right)$ of the $\mathrm{Li}$ vapor based on the ADK theory. The peak fractional ionization is calculated by rewriting Eq. 1 into a more convenient form which depends only on the local electric field magnitude (in units of $\mathrm{GV} / \mathrm{m}$ ) and the ionization energy (in units of $\mathrm{eV}$ )

$$
\begin{aligned}
W_{A D K}\left[\mathrm{~s}^{-1}\right] \approx & 1.52 \times 10^{15} \frac{4^{n^{*}} \varepsilon_{0}}{n^{*} \Gamma\left(2 n^{*}\right)}\left(20.5 \frac{\varepsilon_{0}^{3 / 2}}{E}\right)^{2 n^{*}-1} \\
& \times \exp \left(-6.83 \frac{\varepsilon_{0}^{3 / 2}}{E}\right)
\end{aligned}
$$

where $n^{*} \approx 3.69 Z / \varepsilon_{0}^{1 / 2}[3]$. The ionization rate is integrated numerically for the local electric field of the bunch, neglecting the focusing effects due to the ion column. The 
TABLE III: Lithium Threshold Conditions

\begin{tabular}{r|lr|lr}
\hline \hline & Data & & Calculation & \\
\hline $\mathrm{Li}-\Delta \sigma_{r}$ & $N$ (scaled) & $0.68 \times 10^{10}$ & $E_{\text {peak }}$ & $5.25 \mathrm{GV} / \mathrm{m}$ \\
& $\sigma_{r}$ & $21 \mu \mathrm{m}$ & $F_{\text {peak }}$ & $54.30 \%$ \\
& $\sigma_{z}$ & $32 \mu \mathrm{m}$ & & \\
$\mathrm{Li}-\Delta N$ & $N$ (scaled) & $0.51 \times 10^{10}$ & $E_{\text {peak }}$ & $5.08 \mathrm{GV} / \mathrm{m}$ \\
& $\sigma_{r}$ & $20 \mu \mathrm{m}$ & $F_{\text {peak }}$ & $27.67 \%$ \\
& $\sigma_{z}$ & $26 \mu \mathrm{m}$ & & \\
$\mathrm{Li}-\Delta \sigma_{z}$ & $N$ (scaled) & $0.77 \times 10^{10}$ & $E_{\text {peak }}$ & $4.08 \mathrm{GV} / \mathrm{m}$ \\
& $\sigma_{r}$ & $15 \mu \mathrm{m}$ & $F_{\text {peak }}$ & $1.64 \%$ \\
& $\sigma_{z}$ & $66 \mu \mathrm{m}$ & \multicolumn{2}{c}{} \\
\hline \hline
\end{tabular}

maximal value of that calculation is defined as the peak fractional ionization.

Although the calculated peak factional ionization varies from less than $5 \%$ up to $50 \%$, the threshold fields as measured in the experiment are in reasonable agreement with the conditions expected from the ADK approximation. Variations in the threshold field conditions between the three experiments are attributed to errors in the beam parameter measurements. Considering that the ionization rate has an exponential dependance on the electric field strength, small errors in determining the beam parameters can significantly alter the peak factional ionization calculation. The ADK threshold conditions are within the limitations of the experiment to accurately determine the incoming beam parameters.

\section{SIMULATIONS FOR Li}

This section will compare the experimental results with simulation, which is necessary to analyze the field ionization threshold in context of the beam's energy loss due to the plasma wake production. For the beam and plasma parameters produced in these experiments, the use of numerical codes is required to simulate both field ionization effects, once the beam's radial electric field surpasses a certain threshold, and the resulting plasma wake field physics. The 2-D Object-Oriented Particle-In-Cell code, OOPIC, fulfills those requirements and is a commercially available code developed through the Tech-X Corporation and the University of California at Berkeley [12]. In particular, the peak and average energy loss of the beam as calculated by the simulations are compared to the $\mathrm{Li}$ data. Providing the simulations adequately describe the experimental conditions, they will offer additional insight into the secondary ionization effects.

OOPIC also uses the ADK model to determine the probability rate for ionization. The ADK model in OOPIC has been validated via a direct comparison with experimental data from the l'OASIS laboratory $[13,14]$. The field ionization model was confirmed for the first and sec- ondary ionization of helium by comparing the simulation to measurements of the dependance of the blue-shifted wavelength on the laser pulse length.

Although the code has been benchmarked by experiment, the simulation only approximates the experimental conditions presented in this paper. For example, the incoming particle beam can only have a Gaussian or polynomial charge distribution along the radial and longitudinal components. The vapor profile must also be approximated, since the code assumes sharp vapor boundaries rather than a continuous transition region, which is associated with heat-pipe ovens. Additionally, the incoming beam has an effective emittance of zero, as no angular divergence is calculated for the beam and the simulation is unable to include a correlated energy spread in the incoming beam; however, this last effect does is not expected to alter the results presented here. The simulation code does include the secondary effects, such as focusing, but does not include the synchrotron radiation energy loss, which can be significant when a large transverse beam ionizes and traverses a high-density vapor.

In the following three sections, the number of simulation particles used to describe the experiment varied in order to optimize the resolution of the simulation and minimize the CPU time. The total number of simulation particles ranged from 4,000 to about 12,000, depending on the incoming beam's parameters. Since the input for OOPIC assumes a Gaussian distribution and the lowcurrent wings do not significantly affect the ionization threshold, only the center of the Gaussian fit from the longitudinal profiles produced by LITRACK is used, refer to Fig. 2. The total beam charge is then scaled in the OOPIC simulations so that the peak current is the same for the LITRACK results.

Because OOPIC assumes a sharp boundary for the vapor, the entire vapor column, including the transition regions, is approximated in the simulation code. For example, if the oven has as a vapor column of $6 \mathrm{~cm}$ long and an $8 \mathrm{~cm}$ transition regions on either side, the oven profile is approximated as a $14 \mathrm{~cm}$ column at full density, with sharp boundaries, since the energy loss of the bunch is linear with plasma density.

\section{A. Changing Transverse Beam Size}

While holding the charge and bunch length constant, eight separate OOPIC runs varied the transverse beam size from $25 \mu \mathrm{m}$ down to $10 \mu \mathrm{m}$. The results from the eight simulation runs are shown in Fig. 11. The simulation results are converted from beam size to waist location using previous measurements with a wire scanner along the $x$-component as a guide [4]. The OOPIC results are equivalent to the waist location of around $-32 \mathrm{~cm}$ to 0 in the data.

At the smallest beam size, the simulation calculated a peak energy loss of $735 \mathrm{MeV}$, whereas the data showed an energy loss of approximately $800 \mathrm{MeV}$. The simulated 


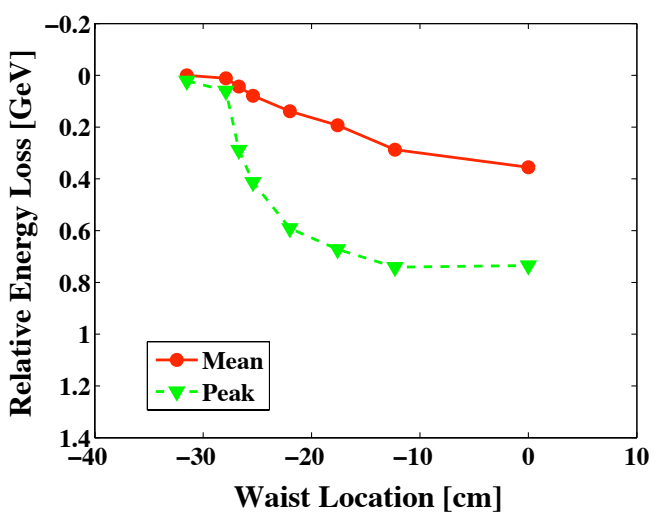

FIG. 11: Changing Beam Size (Li) - The average and peak energy loss results from OOPIC.

average energy loss for the smallest beam size was only $355 \mathrm{MeV}$, which is much smaller than the average loss of $600 \mathrm{MeV}$ measured in the experiment.

Since the simulation does not include any energy losses associated with synchrotron radiation, those effects are calculated for the beam parameters by using the method described in Kostyukov's paper [15]. The energy loss of an electron per unit distance traveled due to synchrotron radiation, averaged over one full cycle of oscillation, is approximately:

$$
Q[\mathrm{MeV} / \mathrm{cm}] \simeq 1.5 \times 10^{-45}\left(\gamma n_{p}\left[\mathrm{~cm}^{-3}\right] \sigma_{r}[\mu \mathrm{m}]\right)^{2},
$$

The oven profile is approximated as a step function where the transition regions are at half density, $1.5 \times 10^{16} \mathrm{~cm}^{-3}$, and the center column at full density, $3 \times 10^{16} \mathrm{~cm}^{-3}$. For a bunch with a peak radial beam size of $25 \mu \mathrm{m}$ traversing a plasma with a $10 \mathrm{~cm}$ center column and $7 \mathrm{~cm}$ transition regions, the energy loss averaged over the full oscillation due to synchrotron radiation would be approximately $35 \mathrm{MeV}$. This additional energy loss due to the betatron oscillation is only an approximation, since it assumes a constant beam size and does not include any variation due to the focusing effects; however, these losses are minimal nonetheless.

As is seen in Fig. 11, the threshold for ionization is crossed for a beam waist location of approximately $30 \mathrm{~cm}$ in the simulation, which is in excellent agreement with that observed in the data (Fig. 4). Considering the cases discussed in this paper were all around threshold conditions, none should produce sufficiently high fields to ionize the second electrons. The OOPIC simulation confirmed those expectations and also indicated that no secondary electrons were created by collisions with neutrals.

\section{B. Changing Charge}

Five separate OOPIC runs varied the charge from $0.65 \times 10^{10}$ to $1.43 \times 10^{10}$ particles per bunch. At the maximum charge case, the simulation calculates a peak en-

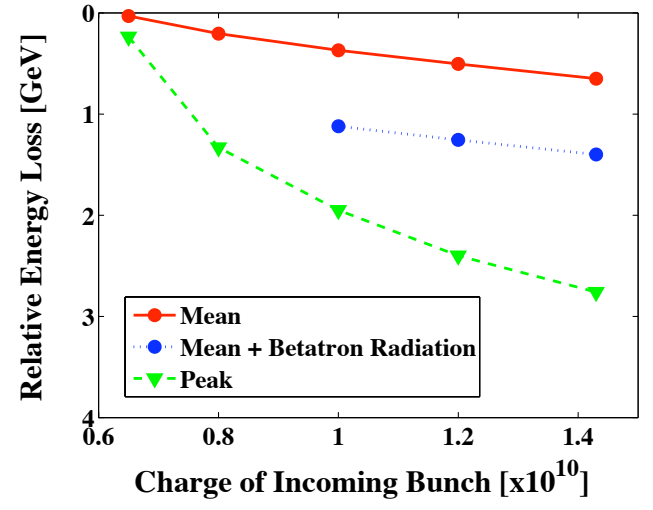

FIG. 12: Changing Charge (Li) - The average and peak energy loss results from OOPIC.

ergy loss of around $3 \mathrm{GeV}$, while the measured peak energy loss was approximately $4 \mathrm{GeV}$. For the average energy loss at the maximum charge, the simulation calculates $757 \mathrm{MeV}$ which is far below the roughly $1.5 \mathrm{GeV}$ measured.

Once again the energy loss associated with the synchrotron radiation needs to be considered. As opposed to the previous data set, the incoming beam's transverse size is not only large $(20 \mu \mathrm{m})$, but the $\mathrm{Li}$ vapor is particularly dense $\left(2 \times 10^{17} \mathrm{~cm}^{-3}\right)$ so the energy loss as a result of betatron oscillations is non-negligible.

Repeating the same calculation from the previous section, the average energy loss due to betatron radiation would be approximately $750 \mathrm{MeV}$ for this data set. Including the additional energy loss to the simulation's average energy loss of $757 \mathrm{MeV}$ results in a total energy loss of $1.5 \mathrm{GeV}$ at the highest charge, which is in agreement with the measured value.

\section{Changing Bunch Length}

In the case of changing bunch length, five separate OOPIC runs varied the bunch length from $65 \mu \mathrm{m}$ down to $25 \mu \mathrm{m}$ and the simulation results are plotted in Fig. 13.

Looking at the minimum bunch length case, the simulation calculates a peak energy loss of $1.1 \mathrm{GeV}$, which is in good agreement with the measured peak energy loss of $1.2 \mathrm{GeV}$. For the average energy loss at the minimum bunch length, the simulation calculated $419 \mathrm{MeV}$, whereas the experiment measured $800 \mathrm{MeV}$. In this case, the energy loss as a result of betatron oscillations has a minimal effect, only $20 \mathrm{MeV}$, because the transverse beam size is relatively small $(15 \mu \mathrm{m})$ and the plasma density is much lower $\left(3 \times 10^{16} \mathrm{~cm}^{-3}\right)$. 


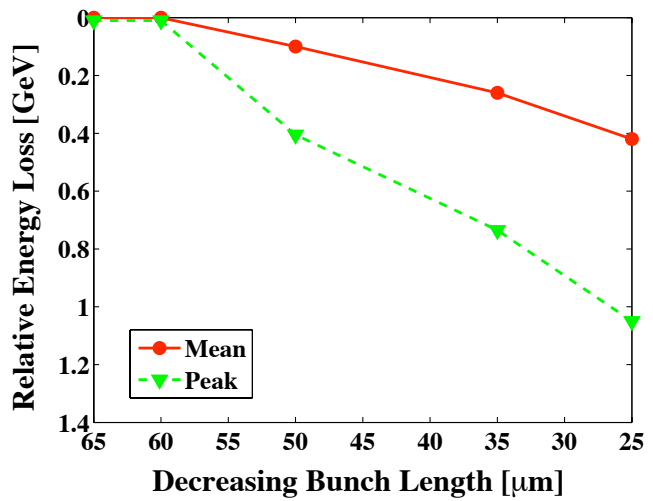

FIG. 13: Changing Bunch Length (Li) - The average and peak energy loss results from OOPIC.

\section{Data and Simulation Comparison}

Since OOPIC assumes a Gaussian beam with the same characteristics as the center peak of the LITRACK distribution, we would expect the peak energy loss of the simulation to be fairly accurate when compared with the experimental data. When a significant discrepancy exists between the data and simulation in the peak energy loss, as in the case of changing charge, it is due to synchrotron radiation effects which are not included in the simulation.

Although the Gaussian approximation does not alter the simulation's accuracy with respect to the peak energy loss, it does adversely affect the average energy loss. For example, the incoming beam in both the changing transverse beam size and bunch length cases has a long low-current tail and those particles are neglected in the simulation's Gaussian approximation. Because the tail particles are decelerated in the data but ignored in the simulation, the calculated average energy loss is much less than that which is measured. In the case of the changing charge, the incoming beam has a long low-current head rather than a tail (Fig. 2(B)), so the difference in the average energy loss is accounted for by losses due to synchrotron radiation.

\section{CONCLUSIONS}

We have demonstrated that by independently varying the transverse beam size, charge or bunch length of the incoming electron beam, the self-fields of the beam are controlled to be above or below the threshold for field ionization of lithium as observed in the experiment through the energy loss of the electron beam. The measurements performed with lithium are consistent with first order calculations of the ADK approximation and is in agreement with the simulation code, OOPIC, within the limitations of experiment and simulation. Additional experiments that varied in the incoming beam's bunch length also showed field ionization effects in xenon and nitric oxide by again observing the beam's energy loss.

Field ionization is an essential ingredient for producing a long, high-density plasma. A self-ionized plasma has already demonstrated the acceleration of particles by $3 \mathrm{GeV}$ [1]. This is a crucial step in the progression towards future high-energy plasma acceleratosrs.

\section{Acknowledgments}

The authors would like to thank Dr. Peter Tsou of JPL for the aerogel and Rasmus Ischebeck for his assistance with LITRACK. This work supported by Department of Energy Contracts No. DE-AC02-76SF00515 (SLAC), No. DE-FG03- 92ER40745, No. DE-FG0398DP00211,No. DE-FG03-92ER40727, No. DE-AC0376SF0098 and National Science Foundation Grants No. ECS-9632735, No. DMS-9722121, and No. PHY0078715 .
[1] M. J. Hogan et al., Phys. Rev. Lett. 95, 054802 (2005).

[2] S. Lee et al., Physical Review Special Topics - Accelerators and Beams 5 (2002).

[3] D. L. Bruhwiler, D. A. Dimitrov, J. Cary, E. Esarey, W. Leemans, and R. Giacone, Physics of Plasmas 10, 2022 (2003).

[4] C. L. O'Connell, Ph.D. thesis, Stanford University (2005).

[5] M. V. Ammosov, N. B. Delone, and V. P. Krainov, Sov. Phys. JETP 64, 1191 (1986).

[6] C. D. Barnes et al., in "IEEE Particle Accelerator Conference" (Knoxville, TN, 2005), pp. 1856-1858.

[7] C. E. Clayton et al., Physical Review Letters 88, 154801 (2002).

[8] P. Muggli et al., IEEE Transactions on Plasma Science 27, 791 (1999).
[9] T. D. G. Walsh, J. E. Decker, and S. L. Chin, Journal of Phys. B: Mol. Opt. Phys. 26 (1993).

[10] T. D. G. Walsh, F. A. Ilkov, J. E. Decker, and S. L. Chin, Journal of Phys. B: Mol. Opt. Phys. 27, 3767 (1994).

[11] K. L. F. Bane and P. Emma, in "IEEE Particle Accelerator Conference" (Knoxville, TN, 2005), pp. 4266-4268.

[12] D. L. Bruhwiler, R. E. Giacone, J. R. Cary, J. P. Verboncoeur, P. Mardahl, and E. Esarey, Physical Review Special Topics: Accelerators and Beams 4 (2001).

[13] W. P. Leemans, P. Catravas, E. Esarey, C. G. R. Geddes, C. Toth, R. Trines, C. B. Schroeder, B. A. Shadwick, J. Van Tilborg, and J. Faure, Phys. Rev. Lett. 89 (2002).

[14] W. P. Leemans, D. Rodgers, P. Catravas, C. G. R. Geddes, G. Fubiani, and E. Esarey, Phys. Plasmas 8 (2001).

[15] I. Kostyukov, S. Kiselev, and A. Pukhov, Phys. of Plasmas 10, 4818 (2003). 